\title{
Structure and function of RNA elements present in enteroviral genomes
}

\author{
Mariola Dutkiewicz ${ }^{\bowtie}$, Aleksandra Stachowiak, Agata Swiatkowska and Jerzy Ciesiołka \\ Institute of Bioorganic Chemistry, Polish Academy of Sciences, Poznań, Poland
}

Enteroviruses are small RNA(+) viruses that encode one open reading frame flanked by two extensive noncoding regions carrying structural RNA regulatory elements that control replication and translation processes. For a long time the central, coding region was thought to remain single-stranded and its only function was supposed to be as the template for polyprotein synthesis. It turned out, however, that the protein coding region also encodes important RNA structures crucial for the viral life cycle and virus persistence in the host cells. This review considers the RNA structures in enteroviral genomes identified and characterized to date.

Key words: Enterovirus, poliovirus, coxsackievirus B3, RNA structure, structural RNA element, cis-acting element

Received: 31 May, 2016; revised: 28 June, 2016; accepted: 12 August, 2016; available on-line: 10 November, 2016

\section{INTRODUCTION}

The Enterovirus genus belongs to the Picornaviridae family, which includes other small non-enveloped RNA(+) viruses with an icosahedral capsid (Racaniello, 2007; Muehlenbachs et al., 2015). Based on molecular and serological analyses, 12 species of enteroviruses have been identified: Enterovirus $A-J$ and Rbinovirus $A-C$. The best characterized member of the enterovirus genus is the poliovirus (PV), a member of Enterovirus $C$ species, which causes poliomyelitis (Mehndiratta et al., 2014; Muehlenbachs et al., 2015). Another enterovirus which is often chosen as a model for molecular research is the heart pathogen coxsackievirus B3 (CVB3), a member of Enterovirus B species, in part because of its similarities with PV in terms of structure and life cycle, while at the same time being much safer to work with.

The viral life cycle lasts 5 to 10 hours, depending on the serotype. Initially, the virus recognizes a specific receptor located on the surface of a host cell and is internalized. Low $\mathrm{pH}$ in the endosomes as well as virus' interaction with co-receptors lead to the release of the viral genetic material. In contrast to DNA viruses, $\mathrm{RNA}(+)$ viruses do not move into the nucleus and viral RNA can be used immediately as a template for translation machinery to begin production of the viral proteins. When virus proteins reach a high enough concentration, the genomic RNA strand is used as a template for replication. Newly synthesized RNAs can go into the next round of replication or they can be packed into virions and released by cell lysis (Garmaroudi et al., 2015).
The viral genome is the most important element in initiation of the host inflammation. This genome is a single-stranded plus RNA strand which is approximately $7400 \mathrm{nt}$ in length, with about $6500 \mathrm{nt}$-long open reading frame encoding a single viral polyprotein (Fig. 1). The 5' end of the viral genome is bound by the VPg protein, whereas at the very 3' end a poly(A) tail is present (Semler, 2004; Racaniello, 2007).

Untranslated regions (UTR), flanking the open reading frame, comprise around $10 \%$ of the entire viral genome. They contain RNA structural elements which play regulatory functions in the viral life cycle. The 5' UTR contains an IRES element (internal ribosome entry site) that is responsible for initiation of cap-independent translation and a cloverleaf structure indispensable for replication. The structurally ordered 3' UTR is involved in replication initiation and viral circularization as well (Semler, 2004; Racaniello, 2007; Liu et al., 2009).

The coding region was originally thought to be single-stranded and its only function to be a template for polyprotein synthesis. As structural RNA features became better understood, it became apparent that they are present in the coding part as well. The region encoding the viral polyprotein is divided into three regions: P1, P2 and P3 (Semler, 2004). Genes in the P1 block express structural proteins, and the P2 part encodes proteins and a cre RNA structure which are both involved in the replication cycle of the virus. The most crucial protein factors, precursor 3CD, proteinase $3 \mathrm{Cpro}^{\mathrm{pro}}$ RNA replicase $3 \mathrm{D}^{\mathrm{pol}}$, protein $3 \mathrm{~A}$ and peptide $\mathrm{VPg}$, are synthesized from the P3 region (Semler, 2004). Recently, several new RNA elements have been discovered within P3, which are also engaged in propagation of the viral cycle or virus persistence in the host cells.

In this review, we present current knowledge regarding structural elements which have been found in the enterovirus genomes (Fig. 1). Some of these motifs are common for all species (cloverleaf, IRES, cre, $\mathrm{Y}$ ), while others are specific for particular enterovirus types (X, Z, i-RNaseL, 3D-7000, E10). These structural RNA features allow for infection and propagation in order to continue the viral cycle. Moreover, there are also motifs (E10, II',III') found in the enterovirus genomes whose function is still unknown and further research is necessary to reveal the role of these structural elements in the viral life cycle.

e-mail: mariolad@ibch.poznan.pl

Abbreviations: CVB3, coxsackievierus B3; PV, poliovirus; UTR, untranslated region; IRES, internal ribosome entry site; PABP, poly(A)binding protein; $P C B P$, poly $(r C)$-binding protein; RNase $\mathrm{L}$, ribonuclease $L$; hnRNP $C$, heterogeneous nuclear ribonucleoprotein $C ; R F$, replicative form 


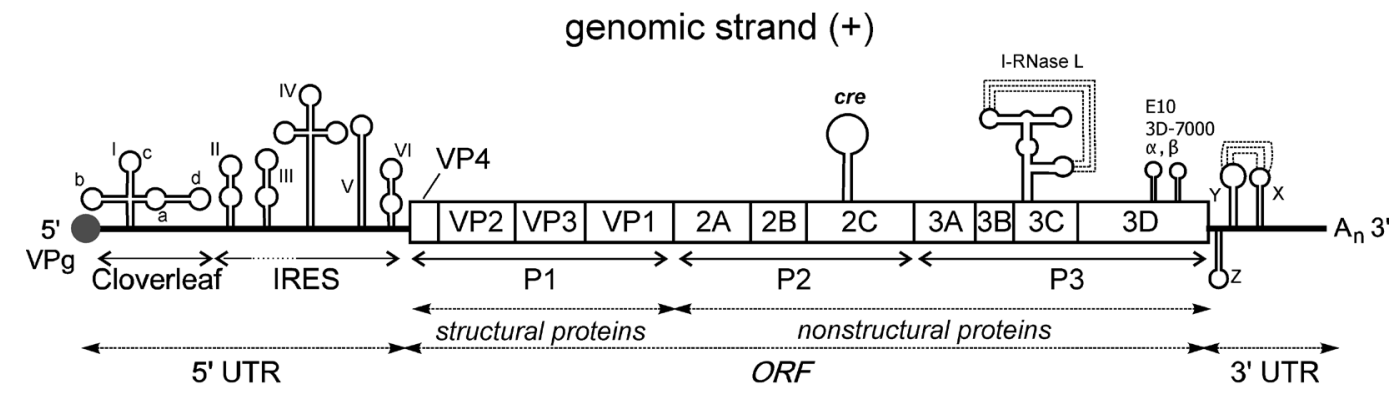

minus strand (-)

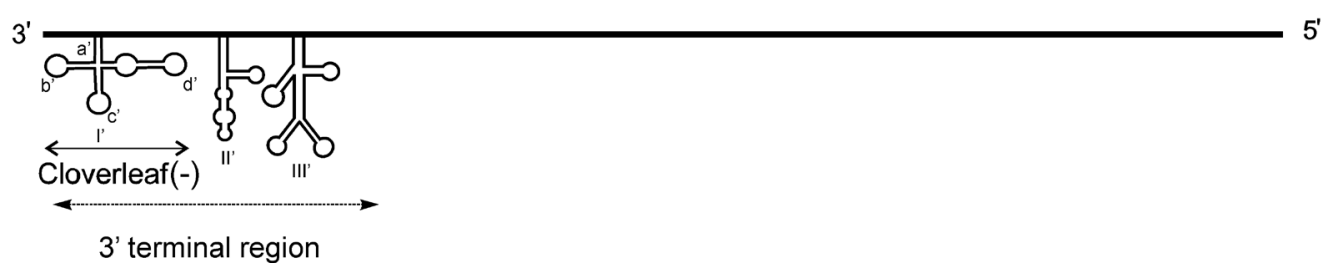

Figure 1. Location of the structural RNA elements and protein coding regions along the enterovirus (PV, CVB) genome.

UTR - untranslated region; IRES - internal ribosome entry site; cre - cis-acting replicative element; i-RNaseL - RNase L inhibitor; E10, 3D7000, a, $\beta$ - structural RNA elements found in the region encoding 3D Pol of poliovirus; X, Y, Z - stem-loop structures; $A_{n}-$ poly $(A)$ tail; Roman letters depict structural RNA domains; lowercase letters depict their subdomains; ORF - open reading frame of the polyprotein, VP1, VP2, VP3, VP4 - capsid proteins; 2A, 2B, 2C, 3A, 3B, 3C, 3D - non-structural proteins; P1 - region encoding structural proteins; P2 and $\mathrm{P} 3$ - regions encoding non-structural proteins; VPg - virally encoded peptide, covalently linked to the 5' end of the genome. For the functions of the viral proteins, please see (Dutkiewicz et al., 2012).

\section{IRES ELEMENTS AND THEIR ROLE IN THE TRANSLATION PROCESS}

With some exceptions, the translation initiation process in eukaryotes is dependent on the $\mathrm{m}^{7} \mathrm{G}$ cap at the 5' end of an mRNA. Enteroviruses are able to completely inhibit cap-dependent translation. Instead, the ribosome is recruited to the viral genomic RNA by an IRES, allowing the genomic RNA to serve as an mRNA. The IRES element is composed of several structural domains connected by single-stranded fragments of RNA present within the $5^{5}$ UTR of the PV genome, which is approximately $750 \mathrm{nt}$ in length. It has been demonstrated that domain II and domains IV-VI comprise an IRES element, which is involved in initiation of the translation process and allows binding of the ribosome to mRNA without a cap-structure. Interestingly, the first IRES element ever described was that of the poliovirus (Pelletier \& Sonenberg, 1988).

The presence of an IRES element within viral RNA means that synthesis of host proteins can be blocked by inactivation of the complex which is responsible for capstructure recognition, and at the same time production of viral proteins proceeds undisturbed. The stable secondary structure of the IRES makes the recruitment of the small $40 \mathrm{~S}$ ribosome subunit possible without the involvement of the cap element. Hydrolysis of GTP allows for association of the large $60 \mathrm{~S}$ subunit of the ribosome and translation can be initiated. The structural features of an IRES are characteristic of cis-regulatory elements which are important for viral translation due to their ability to interact with translation initiation factors and RNA-binding proteins (Filbin \& Kieft, 2009; FernandezMiragall et al., 2009; Lozano \& Martinez-Salas, 2015).

Five types of viral IRES element have been characterized so far. Types: I, II and III are specific for Picornaviridae, type IV, hepatitis $C$ virus-like is present in the genome of some Flaviviridae and an IRES motif identified in aichivirus (Lozano \& Martinez-Salas, 2015). Translation initiation via IRES depends not only on the structural RNA features, but on interactions with specific factors as well (Filbin \& Kieft, 2009). The sequence of an IRES is not conserved among different types, however, all IRES elements contain a tetra-loop GNRA element (where $\mathrm{N}$ means any nucleotide and $\mathrm{R}$ means a purine) with a poly-pyrimidine tract located upstream of the AUG codon (Fig. 2) (Bhattacharyya \& Das, 2005; Balvay et al., 2009; Filbin \& Kieft, 2009). It has been shown that the lack of the GNRA structure results in decrease in the translation efficiency, indicating the role of this motif in viral protein synthesis (Bhattacharyya \& Das, 2005).

In the translation process of viral proteins, besides the canonical translational initiation factors (eIFs), GTP, tRNA and poly(A) tail, there are also trans-elements, initiation translation activation factors (ITAFs) and viral proteases involved (Balvay et al., 2009). In the case of PV, 2-3 hours after infection, the eIF4G factor is cleaved by the viral protease $2 \mathrm{~A}$, disturbing the binding of eIF3 and eIF4E to the ribosomal complex and inhibiting translation of the host proteins. Another viral protease, 3Cpro, inactivates the PABP protein (poly(A)-binding protein). Lack of functional PABP leads to complete inhibition of the cap-dependent translation of the host proteins, giving the virus a nearly complete monopoly of the host cell's translation machinery (Balvay et al., 2009).

\section{RNA STRUCTURES TAKING PART IN REPLICATION PROCESS OF ENTEROVIRUSES}

Eukaryotes replicate their genetic material in the nucleus using a DNA polymerase and other components of the replication complex. Enteroviruses encode their own replicase, RNA-dependent RNA pol- 


\section{Domain IV}

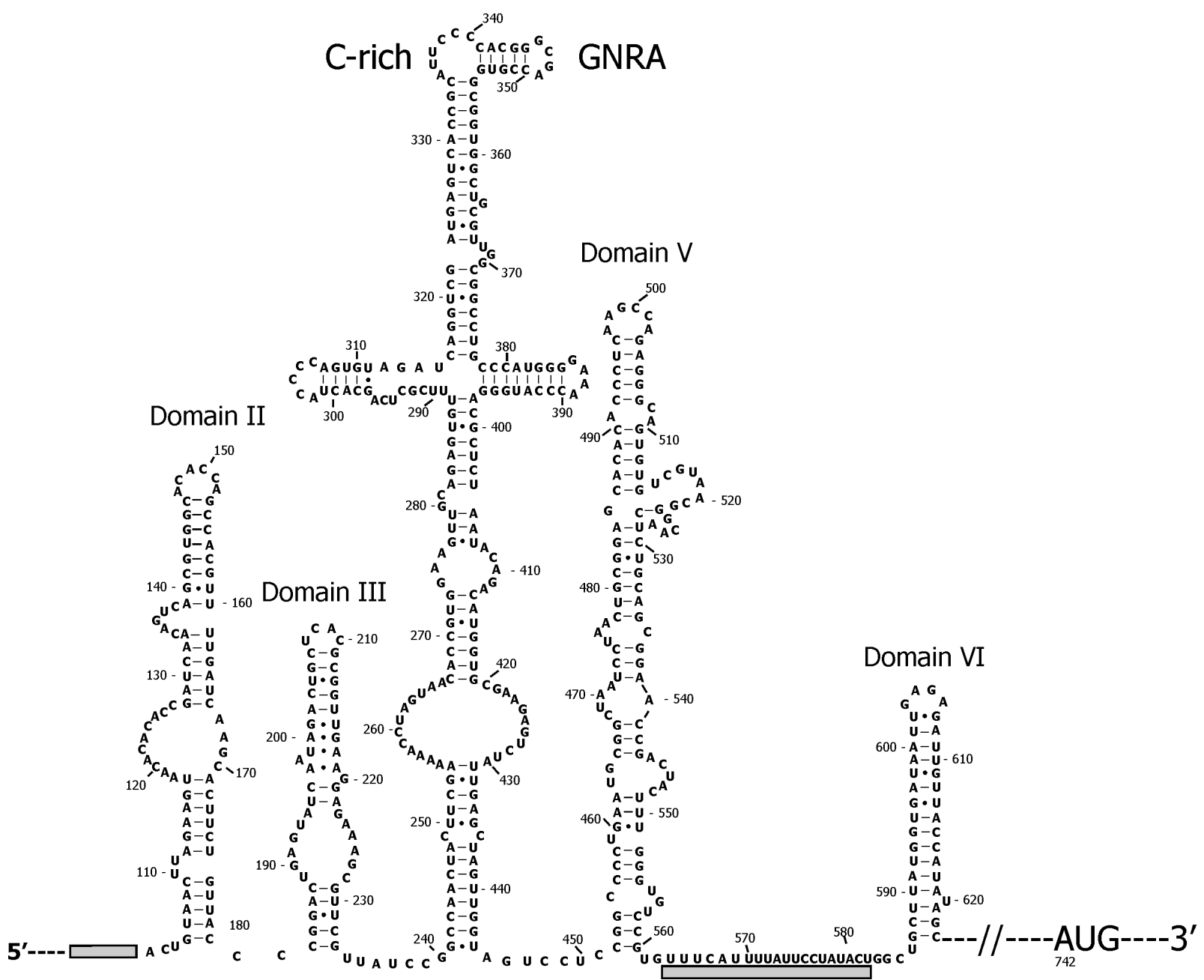

Figure 2. The IRES element type I.

Each of the sequence motifs characteristic for the type I IRES: C-rich sequence, GNRA, polypirymidine stretches (rectangles in the figure), are present in the $5^{\prime}$ UTR of the enteroviral genome. Nucleotide sequence shown is that of coxsackievirus B3. Secondary structure model of domains II-VI adapted from Bailey \& Tapprich (2007).

ymerase, and they replicate in the cytoplasm of the cell. Virus replication starts a few hours after infection and takes place in the membranous vesicles (Steil \& Barton, 2009; van der Linden et al., 2015). During the first step, single-stranded $\mathrm{RNA}(-)$ is synthesized and then $\mathrm{RNA}(+)$ is produced using the minus RNA strand as a template. The newly synthetized $\mathrm{RNA}(+)$ strands can be incorporated into progeny virions or take part in the next round of replication or translation. There are several structural elements encoded in the RNA genome which are essential for enterovirus replication: the cloverleaf motif (also named domain I, oriL) present in the 5' UTR, its complementary structure, cloverleaf $(-)$, located at the 3 ' end of the minus strand, the cre element in the coding part of 2C, the 3' UTR and the poly(A) tail (Liu et al., 2009).

\section{Cloverleaf structure}

All structural RNA elements mentioned above, particularly the cloverleaf, are involved in the first step of the synthesis of the minus strand. The cloverleaf is especially important for replication, but also has a tuning role in translation (Vogt \& Andino, 2010). Presence of the cloverleaf motif is necessary for uridylylation of $\mathrm{VPg}$, which plays a role as a protein starter in the replication of both, the plus and minus RNA strands (Barton et al., 2001; Sharma et al., 2009).

The cloverleaf structure is highly conserved among all enteroviruses. It is composed of four stem-loops, SL a-d (Fig. 3). Hairpin b and the cytidine-rich sequence adjacent to the cloverleaf interact with the cellular PCBP protein (poly $(\mathrm{rC})$-binding protein) which is also known as hnRNP E (Vogt \& Andino, 2010). Disturbance of these interactions inhibits viral replication (Sharma et al., 2009). Stem-loop d binds the viral proteins, 3C and $3 \mathrm{CD}^{\text {pro }}$, and its interaction with $3 \mathrm{CD}^{\text {pro }}$ is required for replication of the plus and minus RNA strands (Claridge et al., 2009; Sharma et al., 2009). The replication complex is formed by interactions between the cloverleaf element with PCBP and viral 3CDpro (Steil \& Barton, 2009). Mutations within the cloverleaf structure cause a lower efficiency of $\mathrm{RNA}(+)$ synthesis. Moreover, stem-loop "a" plays a crucial role in efficient production of the plus strand RNA (Vogt \& Andino, 2010).

\section{The 3'UTR}

Besides the cloverleaf element, other structural features, such as the oriR present at the 3' terminus of the 

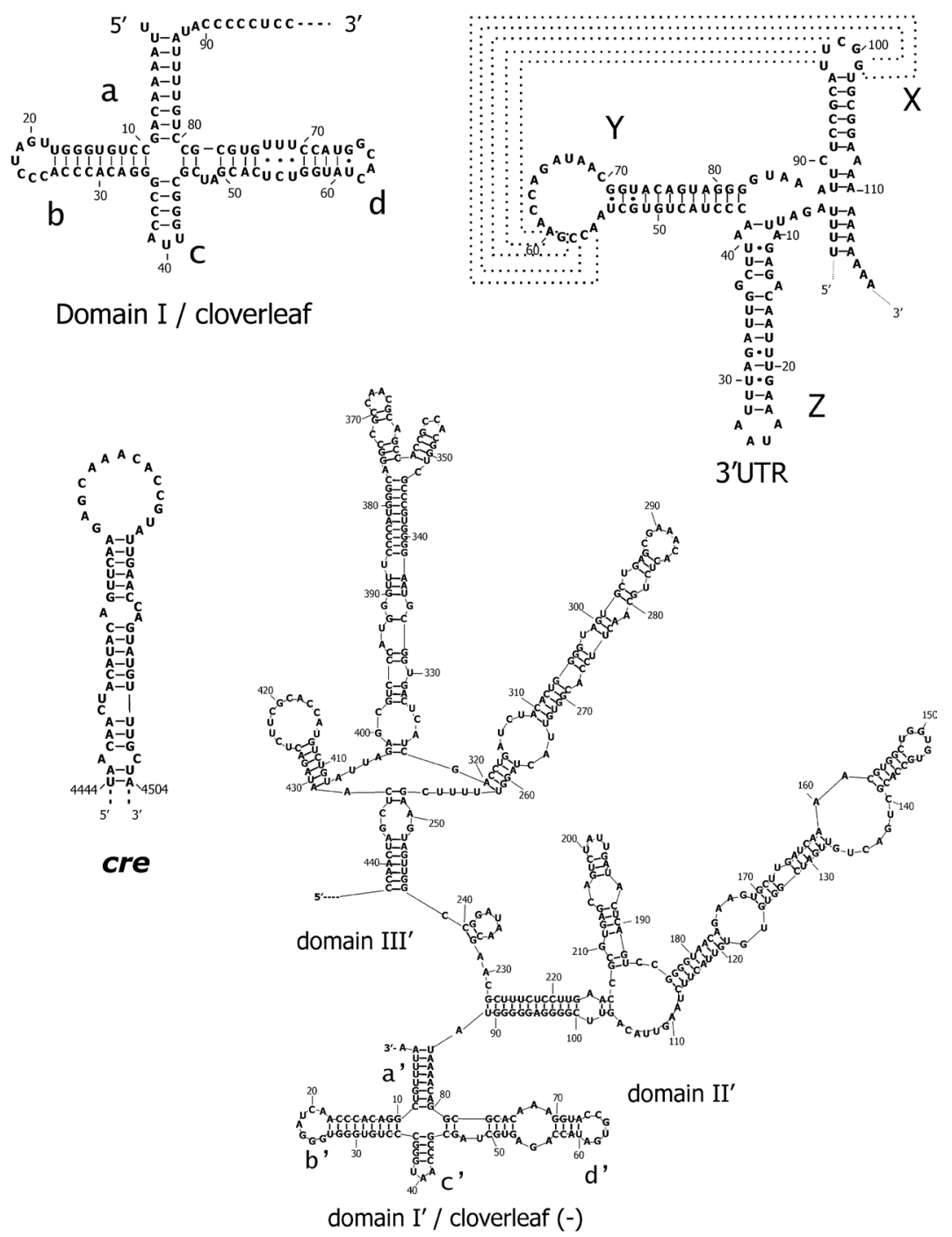

\section{3 '-terminal region of the minus strand}

Figure 3. RNA structures taking part in replication process of enteroviruses.

Domain I/cloverleaf structure is present in the $5^{\prime}$ UTR of enteroviruses. Nucleotide sequence is that of coxsackievirus B3. The stem-loops: a, b, c and d are marked (Sharma et al., 2009); Kissing interactions (dotted lines in the figure) in the 3' UTR are present in all enterovirus genomes except for the rhinoviruses (Zoll et al., 2009). The interaction between loop X and $Y$ forms a kissing-like pseudoknot. Hairpin $Z$ has been found only in Enterovirus B. The nucleotide sequence of coxsackievirus B3 is shown; The cre element structure with nucleotide sequence of poliovirus 1 (Goodfellow et al., 2000); Secondary structure model of the 3' terminal region of the minus strand of coxsackievirus B3 (Dutkiewicz et al., 2014). Domains I'-III' and subdomains: a'd' are marked.

viral RNA genome, are important for the replication process. The oriR is located within the 3' UTR and is composed of several stem-loop motifs. There are the following domains: $\mathrm{X}$ and $\mathrm{Y}$ in the Enterovirus $C$ genomes; $\mathrm{X}$, $\mathrm{Y}$ and $\mathrm{Z}$ domains in the Enterovirus $B$ genomes (Fig. 3), and only the $\mathrm{Y}$ domain in the RNA of rhinoviruses. Mutations in the $\mathrm{X}$ and $\mathrm{Y}$ motifs result in a delay of PV RNA synthesis and complete inhibition of CVB3 replication (van Ooij et al., 2006a, 2006b; Zoll et al., 2009). Interaction of the $\mathrm{X}$ and $\mathrm{Y}$ domains forms a tertiary structure. This interaction was described for the first time in 1992 and was named the "kissing interaction" (Fig. 3). Recently, Zoll and coworkers (2009) described it as a "kissing-like pseudoknot". Most of the nucleotides located in the loops of the $\mathrm{X}$ and $\mathrm{Y}$ domain are engaged in formation of this interaction. In the case of all enteroviruses, except rhinoviruses, six pairs of nucleotides are required. Site-directed mutagenesis has shown that these interactions are crucial for viral RNA synthe- sis, whereas they are not required for viral infection. The relationship between the structure and function of oriR is determined by the distance between the $\mathrm{X}$ and $\mathrm{Y}$ domains. The length of both helices is the same in most enteroviruses. Moreover, the spatial conformation of the domains seems to be important. Mutations in one domain can be compensated for by mutations in the other domain (Zoll et al., 2009).

The presence of a 20-150 nt-long poly(A) tail at the terminus of the 3' UTR, results from the presence of poly $(\mathrm{U})$ in the replicative intermediate (Steil \& Barton, 2009). It means that it is genetically encoded, in contrast to the poly(A) tail of cellular mRNA, which is invariably added by the poly(A) polymerase. The poly(A) tail influences the efficiency of translation and the plus and minus strand synthesis via interaction with cellular PABP. The $\operatorname{poly}(\mathrm{A})$ tail is used as a template for $\mathrm{VPg}$ uridylylation processing by viral polymerase 3Dpol. Uridylyled $\mathrm{VPg}$ binds to the 3' end of the virus RNA and acts as a 
signal for initiation of replication. It is worth mentioning that there are two forms of the $\mathrm{VPg}$ proteins: $\mathrm{VPg}$ and $\mathrm{VPgpUpU}_{\mathrm{OH}}(\mathrm{VPg}$ covalently linked to two uridines by phosphodiester bonds between tyrosine and uridine residues). Both forms function as primers for the PV RNA replication (Paul et al., 1998).

A ribonucleoprotein (RNP) complex formed around the 5'-cloverleaf RNA structure of PV interacts with the PABP bound to the 3'-poly $(\mathrm{A})$ tail, thus linking the ends of the viral RNA and circularizing it (Barton et al., 2001). Formation of this circular RNP complex is required for initiation of the synthesis of both RNA strands. Synthesis of $\mathrm{RNA}(-)$ is completed when the replication complex reaches the 5' end of the genomic strand. Both RNA strands are formed into heteroduplex RF (replicative form), until the next cycle of replication begins.

\section{The cre element}

A well-characterized structure, the cre element (cisacting replication element) is located in the $\mathrm{P} 2$ region of the viral genome (P1 in some rhinoviruses) and it is thought to be involved in VPg uridylylation (Steil \& Barton, 2009). The cre element is highly conserved among enteroviruses. It is folded into a hairpin structure with a 14-nucleotide loop rich in adenosine residues. The loop of the cre hairpin contains a sequence conserved in all enteroviruses: (R1NNNA5A6R7NNNNNNR14) (Cordey et al., 2008; Liu et al., 2009). The conserved sequence: AAAUG for CVB3, AAACA for PV, is present in the loop of the cre hairpin (Fig. 3) (Paul et al., 2000; van Ooij et al., 2006c). The first two adenosine residues are indispensable for the covalent linkage of UMP nucleotides to the VPg protein by the viral polymerase. It has been shown that disruption of the cre sequence and/or structure results in a decrease in the efficiency of the synthesis of the poliovirus plus-strand RNA. In contrast, the cre element is engaged in both steps of replication in case of the coxsackievirus B3 (van Ooij et al., 2006c).

The number of the plus RNA strands is always greater than the number of the minus strands in an infected cell. The ratio of the plus strands to the minus strands is approximately 40:1 for poliovirus (Giachetti \& Semler 1991; Novak \& Kirkegaard 1991). One proposed explanation is based on the observation that replication of $\mathrm{RNA}(+)$ via cre is much more effective than synthesis of RNA(-) due to $\mathrm{VPg}$ uridylylation of the poly(A) template (Paul et al., 2000). Presumably, the VPg uridylylation is responsible for differential production of the plus and minus RNA strands, which reflects the need of the virus for different numbers of each. While $\mathrm{RNA}(-)$ only serves as a template to generate new RNAs, the RNA(+) strand is involved in many events of the viral life cycle, such as translation, replication and virion assembly.

\section{The 3' terminal region of CVB3 minus-strand}

The second step of viral replication requires the cloverleaf structure present at the 5' UTR, the cre element and a short sequence fragment, $10 \mathrm{nt}$ in length, at the 3 ' end of the minus strand. Additionally, viral proteins: 3CD, 3Dpol, 2C and hnRNP C (heterogenous nuclear ribonucleoprotein $\mathrm{C}$ ) are involved in this process. After formation of the replication complex at the 3 ' terminus of $\mathrm{RNA}(-)$, the synthesis of new $\mathrm{RNA}(+)$ is initiated by the 3Dpol polymerase (Steil \& Barton, 2009). It has been shown for the poliovirus that two host proteins, p36 (hnRNP C) and p38, as well as two viral peptides, $2 \mathrm{C}$ and $2 \mathrm{BC}$, interact with this 3'-terminal region of the minus strand (Banerjee et al., 1997; Banerjee \& Dasgupta, 2001a, b; Roehl \& Semler, 1995). Based on computer structure prediction, a cloverleaf(-) motif has been proposed to be formed at the 3 ' end of the minus strand of poliovirus. However, to date, detailed experimental analyses of the 3' terminal part of $\operatorname{RNA}(-)$ have been only performed for coxsackievirus B3. The 3' terminal region of the minus-strand of CVB3, around $450 \mathrm{nt}$ in length, is composed of three domains: I', II', III' (Fig. 3) (Dutkiewicz et al., 2014). Domain I' is folded into a cloverleaf-like structure which resembles the structure present at the 5' UTR. This structural element is also comprised of four stem-loops (a', b', c' and d') which have been characterized by structural probing. Domain II', rich in the GU repeats, is composed of two hairpins. However, structural analyses revealed that the smaller hairpin is less thermodynamically stable. Domain III', $200 \mathrm{nt}$ in length, is folded into three structural motifs which are characterized by the presence of stem-loop features and internal loops (Fig. 3) (Dutkiewicz et al., 2014).

What is the role of cloverleaf $(-)$ at the 3 ' end of the minus RNA strand? This highly conserved structure might function during CVB3 replication via interactions with proteins which are analogous to proteins binding to the poliovirus RNA. Since there are strong structural similarities between PV and CVB3, it is possible that in the CVB3 replication the same cellular and viral proteins are engaged in an analogous way. The presence of the cloverleaf $(-)$ structure has a positive impact on the binding of the viral protein $2 \mathrm{C}$ and precursor $2 \mathrm{BC}$ during $\mathrm{RNA}(+)$ synthesis (Banerjee et al., 1997; Banerjee and Dasgupta, 2001a,b). Protein 2C is an NTPase that contains two regions of RNA-binding activity. The $2 \mathrm{C}$ binding is dependent on the presence of the intact sequence 5'-UGUUUU-3' of stem a' and occurs only when it is embedded in a double-stranded structure, like that present in the cloverleaf motif (Fig. 3) (Banerjee et al., 1997). The binding of $2 \mathrm{BC}$ requires the intact stem b' of the cloverleaf(-) and its special spatial orientation to stem a' (Banerjee and Dasgupta, 2001a,b). The interactions described above have been characterized for the poliovirus, however they seem to be highly probable for coxsackievirus B3 as well. The sequence of stem a' and the secondary structure of stem-loop b' of CVB3 closely resemble that found in the PV virus. Protein 2C also seems to be highly conserved among enteroviruses and may play the same role in both viruses. Motifs a' and b' are located very close to each other in a reverse-parallel orientation (Dutkiewicz et al., 2014). A possible function of such interactions for $\mathrm{RNA}(+)$ synthesis is anchoring of the minus viral strand to the membrane vesicles, where the replication process occurs. Additionally, due to the proposed NTPase activity of protein $2 \mathrm{C}$, it might help to unwind the double-stranded stem a' to make the 3 ' end of the minus strand more accessible to other members of the replication complex. One such member might be hnRNP C protein, which binds to the oligo(U) or poly(A) fragments preferentially in single-stranded regions. Since the stability of stem a' is relatively low, it could be a potential binding site for hnRNP C, as has been shown for the poliovirus (Brunner et al., 2005; Ertel et al., 2010; Li \& Nagy, 2011).

Besides the role of structures present within the 3' terminus of the replicative minus strand in protein interactions, they might act to prevent re-hybridization with complementary plus RNA strand during the synthesis of viral genomes. Presumably, they may allow for the repeated association of the replication complex with the same minus strand and synthesis of up to six nascent RNA strands from a single template, which is still partly 


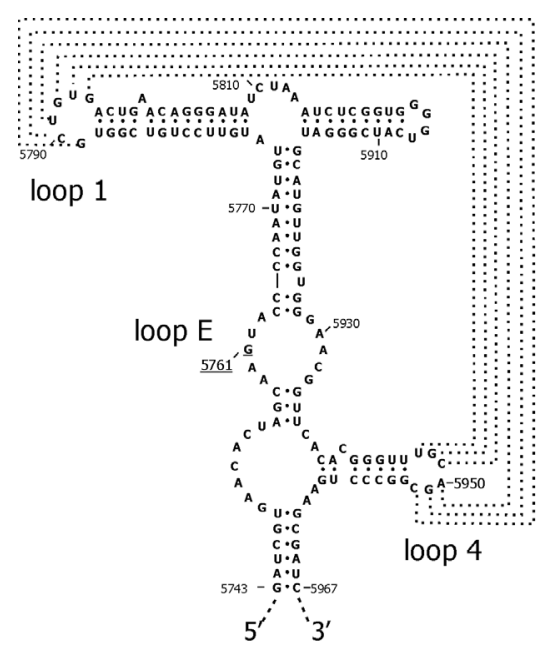

i-RNaseL

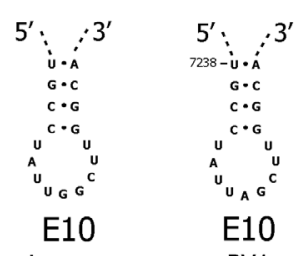

enterovirus consensus PV1

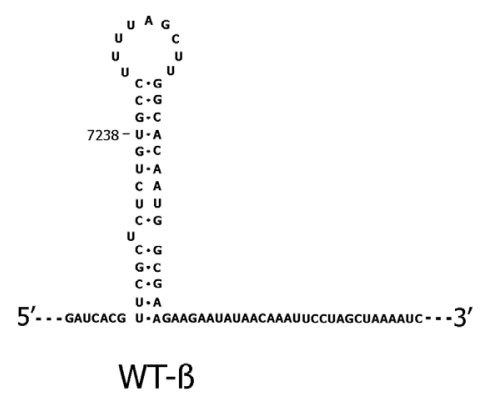

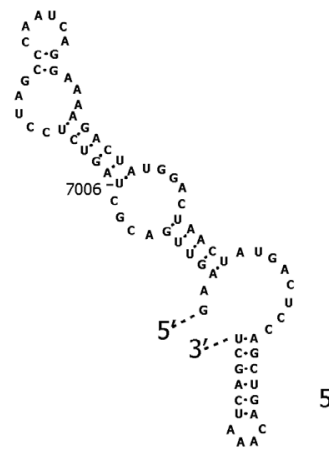

WT- $\alpha$

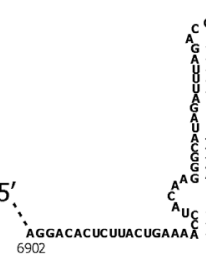

A902

(20)

Figure 4. Structural RNA elements found in P3 region of poliovirus 1.

i-RNaseL - RNase L inhibitor encoded by the 3 C region of Enterovirus C (Han et al., 2007). Dotted lines mark kissing-interactions. Guanosine 5761, crucial for the inhibitory function, is underlined; Newly discovered structural RNA elements in region encoding 3D Pol of poliovirus 1: Hairpin E10 (Witwer et al., 2001), is a part of structure WT- $\beta$ proposed by Song et al. (2012) and structure WT-a (Song et al., 2012) overlaps extensively with the 3D-7000 element characterized by Burrill et al. (2013).

engaged in formation of heteroduplex RF (Sean \& Semler, 2008).

\section{INHIBITION OF RNASE L BY A STRUCTURAL RNA ELEMENT ENCODED IN THE 3C REGION}

Ribonuclease L (RNase L) is one of the key effector enzymes involved in an interferon pathway, whose main role is to defend the host against viruses and other pathogens (Chakrabarti et al., 2011; Jackowiak et al., 2011). The antiviral activity of RNase $\mathrm{L}$ comes from at least two different mechanisms. One of them involves the degradation of viral RNA, and the second promotes cell apoptosis (antiproliferative activity) (Han et al., 2007). RNase L is expressed in a variety of cells as a latent enzyme in its basic, monomeric form. Activation via dimerization occurs after accumulation of viral RNA that triggers an interferon response. Interferon, as well as dsRNA fragments, induce activity of the 2'-5' oligoadenylate synthetase (2-5 OAS) and accumulation of 5' phosphorylated 2'-5' oligoadenylans occurs. This unique 2'-5' A activator binds to ankyrin residues present at the $\mathrm{N}$-terminus of the $\mathrm{RNase} \mathrm{L}$ sequence, inducing a conformational change of the enzyme that leads to its dimerization. As a result, cellular RNase $\mathrm{L}$ is activated and it is able to preferentially digest viral RNA, ribosomal RNA and also other types of cellular RNA (Han et al., 2007; Chakrabarti et al., 2011).

Encoded by the 3C gene of the poliovirus, as well as by several coxsackieviruses A from the Enterovirus $C$ genus, a conserved RNA structural element has been found that competitively inhibits the activity of RNase L (Townsend et al., 2008b). This element, named i-RNaseL or RNase L ciRNA, has an extended, branched stemloop structure and consists of hairpins, double-stranded arms, internal loops and minor mismatches (Fig. 4). The motifs essential for RNase L inhibition are: loops 1 and 4, as well as loop E. Especially important for inhibitory activity is the G5761 residue located in the loop E motif. Nucleotide sequences of loops 1 and 4 are complementary to each other, thus enabling the "kissing interaction" which is mandatory for activity of the inhibitor (Townsend et al., 2008a; Townsend et al., 2008b).

How can such an extended RNA structure inhibit activity of RNase L? It binds the active site of the endoribonuclease domain of the enzyme in a competitive manner. Kinetic analysis demonstrated that it binds with much higher affinity to the active site of RNase L than RNase L's normal substrate, phosphorylated 2'-5' oligoadenylans (Townsend et al., 2008a). In the case of Enterovirus B, such RNA structure like i-RNaseL has not 
been found. These viruses also are much more sensitive to RNase L cleavage. Research based on inhibition of the RNase L activity with RNA structural element may help in antiviral drug design against Enterovirus $C$.

\section{THE SEARCH FOR NEW ORDERED RNA STRUCTURES IN THE CODING REGION OF ENTEROVIRAL GENOMES}

The nucleotide sequence encoding enteroviral polyproteins contains not only information about the protein composition, but also secondary RNA structures important at different points of the viral life cycle, like cre or i-RNaseL. A putative presence of other important RNA elements has been investigated. Several algorithms have been developed that search for conserved RNA structures within coding regions of the RNA viruses. Recently, experimental methods have been also developed that enable structural scanning of large RNA fragments, or even whole viral genomes (Burrill et al., 2013).

Based on computer structural predictions and sequence comparison, Witwer et al. (2001) proposed that enteroviruses, with the exception of the rhinoviruses, contain a strongly conserved and thermodynamically determined RNA hairpin motif E10 at the 3' terminal part of the coding sequence [bttp://wmw.tbi.univie.ac.at/ $\mathrm{RN} A /$.]. It is encompassed within the $3 \mathrm{D}$ gene between nucleotide positions 7414 and 7430 of the PV genome (Fig. 4). To date, the E10 hairpin structure has not yet been experimentally confirmed. However, 11 years later another investigation of the polioviral genome lead to the identification of a larger RNA element called $\beta$ that contains hairpin E10 as a smaller motif within itself (Song et al., 2012). The same publication proposed the existence of a one more RNA element: the $\alpha$ element, present upstream of $\beta$ in the 3 '-terminal 450 -ntlong segment of the $3 \mathrm{D}^{\mathrm{pol}}$ coding sequence (nucleotide positions: 6920-7369). Both RNA structures turned out to be functionally redundant. Presence of those elements is important for efficient replication but more detailed information about their role and structure has not been experimentally verified (Song et al., 2012).

More or less at the same time, another research group performed genome-scale RNA structure characterization of PV using the SHAPE method (selective 2' hydroxyl acylation analyzed by primer extension) (Burrill et al., 2013). They searched for new ordered RNA structures in the coding region and found one very interesting RNA element, 3D-7000, that overlaps extensively with the $\alpha$ structure (Fig. 4). Moreover, the importance of this element for the virus was proven. Mutagenesis studies on this structure revealed that it is important for viral kinetics, dynamic RNA synthesis and infectivity. It was suggested that it might interact with the $3 \mathrm{C}$ or $3 \mathrm{CD}$ protein, since some compensatory mutations were found in the $3 \mathrm{C}$ coding region that eliminated the harmful mutations in the 3D-7000 element (Burrill et al., 2013). However, the detailed mechanism of its function has yet to be discovered.

\section{CONCLUDING REMARKS}

This review summarizes current knowledge regarding structural elements which have been found in enteroviral genomes, mainly those of poliovirus 1 and coxsackievirus B3. These elements are especially characteristic for regulatory, untranslated regions (UTRs) of the viral genomes. However, the protein coding region also encodes important RNA structures crucial for the viral life cycle and virus persistence in the host cells. It turns out that the 3 ' terminal region of the minus strand is also able to fold into ordered RNA structures, but their presence in partially dissociated replicative intermediate form (RF) and function is yet to be proven. Further research is necessary to search for new ordered RNA structures which presumably still hide undiscovered in the coding region, as well as to reveal their role in the virus propagation.

\section{Acknowledgements}

This work was supported by the grant within the PARENT-BRIDGE program of the Foundation for Polish Science, co-financed by the European Union Regional Development Fund, No. POMOST/2013-8/5, to M.D. This publication was also supported by the Polish Ministry of Science and Higher Education, under the KNOW program.

The authors thank Erik Wade for careful reading of the manuscript and valuable comments.

\section{REFERENCES}

Bailey JM, Tapprich WE (2007) Structure of the 5' nontranslated region of the coxsackievirus B3 genome: chemical modification and comparative sequence analysis. J Virol 81: 650-668. doi: 10.1128/ JVI.01327-06

Banerjee R, Echeverri A, Dasgupta A (1997) Poliovirus-encoded 2C polypeptide specifically binds to the 3'-terminal sequences of viral negative-strand RNA. J Virol 71: 9570-9578.

Banerjee R, Dasgupta A (2001a) Interaction of picornavirus 2C polypeptide with the viral negative-strand RNA. I Gen Virol 82: 26212627. doi: 10.1099/0022-1317-82-11-2621

Banerjee R, Dasgupta A (2001b) Specific interaction of hepatitis C virus protease/helicase NS3 with the 3'-terminal sequences of viral positive- and negative-strand RNA. J Virol 75: 1708-1721. doi: 10.1128/JVI.75.4.1708-1721.2001

Barton DJ, O'Donnell BJ, Flanegan JB (2001) 5' cloverleaf in poliovirus RNA is a cis-acting replication element required for negative-strand synthesis. EMBO J 20: 1439-1448. doi: 10.1093/emboj/20.6.1439

Bhattacharyya S, Das S (2005) Mapping of secondary structure of the spacer region within the 5'-untranslated region of the coxsackievirus B3 RNA: possible role of an apical GAGA loop in binding La protein and influencing internal initiation of translation. Virus Res 108: 89-100. doi: 10.1016/j.virusres.2004.08.020

Brunner JE, Nguven JHC, Roehl HH, Ho TV, Swiderek KM, Semler BL (2005) Functional interaction of heterogeneous nuclear ribonucleoprotein $\mathrm{C}$ with poliovirus RNA synthesis initiation complexes. J Virol 79: 3254-3266. doi: 10.1128/JVI.79.6.3254-3266.2005

Burrill CP, Westesson O, Schulte MB, Strings VR, Segal M, Andino R (2013) Global RNA structure analysis of poliovirus identifies a conserved RNA structure involved in viral replication and infectivity. J Virol 87: 11670-11683. doi: 10.1128/JVI.01560-13

Chakrabarti A, Jha BK, Silverman RH (2011) New insights into the role of RNase L in innate immunity. J Interferon Cytokine Res 31: 4957. doi: $10.1089 /$ jir. 2010.0120

Claridge JK, Headey SJ, Chow JY, Schwalbe M, Edwards PJ, Jeffries CM, Venugopal H, Trewhella J, Pascal SM (2009) A picornaviral loop-to-loop replication complex. J Struct Biol 166: 251-262. doi: 10.1016/j.jsb.2009.02.010

Cordey S, Gerlach D, Junier T, Zdobnov EM, Kaiser L, Tapparel C (2008) The cis-acting replication elements define human enterovirus and rhinovirus species. RNA 14: 1568-1578. doi: 10.1261/ rna.1031408

Dutkiewicz M, Ojdowska A, Gorska A, Swiatkowska A, Ciesiolka J (2014) The structural and phylogenetic profile of the 3 ' terminus of coxsackievirus B3 negative strand. Virus Res 188: 81-89. doi: 10.1016/j.virusres.2014.03.020

Dutkiewicz MS, Ojdowska A, Smolska A, Dymarek-Babs B, Jasinska T, Ciesiolka J (2012) Molecular mechnisms of genome expression of coxsackievirus B3 that belongs to enteroviruses. BioTechnologia 93: 414-423. doi: 10.5114/bta.2012.46595

Ertel KJ, Brunner JE, Semler BL (2010) Mechanistic consequences of hnRNP C binding to both RNA termini of poliovirus negativestrand RNA intermediates. J Virol 84: 4229-4242. doi: 10.1128/ JVI.02198-09

Fernandez-Miragall O, Lopez de Quinto S, Martinez-Salas E (2009) Relevance of RNA structure for the activity of picornavirus IRES elements. Virus Res 139: 172-182. doi: 10.1016/j.virusres.2008.07.009 
Filbin ME, Kieft JS (2009) Toward a structural understanding of IRES RNA function. Curr Opin Struct Biol 19: 267-276. doi: 10.1016/j. sbi.2009.03.005

Garmaroudi FS, Marchant D, Hendry R, Luo H, Yang D, Ye X, Shi J, McManus BM (2015) Coxsackievirus B3 replication and pathogenesis. Future Microbiol 10: 629-653. doi: 10.2217/fmb.15.5

Giachetti C, Semler BL (1991) Role of a viral membrane polypeptide in strand-specific initiation of poliovirus RNA synthesis. I Virol 65: 2647-2654.

Goodfellow I, Chaudhry Y, Richardson A, Meredith J, Almond JW, Barclay W, Evans DJ (2000) Identification of a cis-acting replication element within the poliovirus coding region. J Virol 74: 4590-4600.

Han JQ, Townsend HL, Jha BK, Paranjape JM, Silverman RH, Barton DJ (2007) A phylogenetically conserved RNA structure in the poliovirus open reading frame inhibits the antiviral endoribonuclease RNase L. J Virol 81: 5561-5572. doi: 10.1128/jvi.01857-06

Jackowiak P, Figlerowicz M, Kurzynska-Kokorniak A, Figlerowicz M (2011) Mechanisms involved in the development of chronic hepatitis C as potential targets of antiviral therapy. Curr Pharm Biotechnol 12: $1774-1780$.

Li Z, Nagy PD (2011) Diverse roles of host RNA-binding proteins in RNA virus replication. RNA Biol 8: 305-315.

Liu Y, Wimmer E, Paul AV (2009) Cis-acting RNA elements in human and animal plus-strand RNA viruses. Biochim Biophys Acta 1789: 495-517. doi: 10.1016/j.bbagrm.2009.09.007

Lozano G, Martinez-Salas E (2015) Structural insights into viral IRESdependent translation mechanisms. Curr Opin Virol 12: 113-120. doi: 10.1016/j.coviro.2015.04.008

Mehndiratta MM, Mehndiratta P, Pande R (2014) Poliomyelitis: historical facts, epidemiology, and current challenges in eradication. Neurohospitalist 4: 223-229. doi: 10.1177/1941874414533352

Muehlenbachs A, Bhatnagar J, Zaki SR (2015) Tissue tropism, pathology and pathogenesis of enterovirus infection. J Pathol 235: 217-228. doi: $10.1002 /$ path.4438

Novak JE, Kirkegaard K (1991) Improved method for detecting poliovirus negative strands used to demonstrate specificity of positivestrand encapsidation and the ratio of positive to negative strands in infected cells. J Virol 65: 3384-3387.

Paul AV, van Boom JH, Filippov D, Wimmer E (1998) Protein-primed RNA synthesis by purified poliovirus RNA polymerase. Nature 393: 280-284.

Paul AV, Rieder E, Kim DW, van Boom JH, Wimmer E (2000) Identification of an RNA hairpin in poliovirus RNA that serves as the primary template in the in vitro uridylylation of VPg. J Virol 74: 10359-10370. doi: 10.1128/JVI.74.22.10359-10370.

Pelletier J, Sonenberg N (1988) Internal initiation of translation of eukaryotic mRNA directed by a sequence derived from poliovirus RNA. Nature 334: 320-325.

Racaniello VR (2007) Picornaviridae: the virus and their replication. In Field's Virology, Knipe HP, Howley DM eds, 5th edn. Lippincott Williams \& Wilkins a Wolter Kulwer Business, 795-838.

Roehl HH, Semler BL (1995) Poliovirus infection enhances the formation of 2 ribonucleoprotein complexes at the 3' end of viral negative-strand RNA. J Virol 69: 2954-2961.
Semler BL (2004) Poliovirus proves IRES-istible in vivo. I Clin Invest 113: $1678-1681$. doi: $10.1172 /$ jci22139

Sharma N, Ogram SA, Morasco BJ, Spear A, Chapman NM, Flanegan JB (2009) Functional role of the 5' terminal cloverleaf in Coxsackievirus RNA replication. Virology 393: 238-249. doi: 10.1016/j. virol.2009.07.039

Sean P, Semler BL (2008) Coxsackievirus B RNA replication: lessons from poliovirus. In Group B Coxsackieviruses. Tracy S, Oberste MS, Drescher K, eds, pp 89-121 Springer, Berlin, Heidelberg.

Song Y, Liu Y, Ward CB, Mueller S, Futcher B, Skiena S, Paul AV, Wimmer E (2012) Identification of two functionally redundant RNA elements in the coding sequence of poliovirus using computer-generated design. Proc Natl Acad Sci US A 109: 14301-14307. doi: $10.1073 /$ pnas. 1211484109

Steil BP, Barton DJ (2009) Cis-active RNA elements (CREs) and picornavirus RNA replication. Virus Res 139: 240-252. doi: 10.1016/j. virusres.2008.07.027

Townsend HL, Jha BK, Han JQ, Maluf NK, Silverman RH, Barton DJ (2008a) A viral RNA competitively inhibits the antiviral endoribonuclease domain of RNase L. RNA 14: 1026-1036. doi: 10.1261/ rna. 958908

Townsend HL, Jha BK, Silverman RH, Barton DJ (2008b) A putative loop $\mathrm{E}$ motif and an $\mathrm{H}-\mathrm{H}$ kissing loop interaction are conserved and functional features in a group $\mathrm{C}$ enterovirus RNA that inhibits ribonuclease L. RNA Biol 5: 263-272.

van der Linden L, Wolthers KC, van Kuppeveld FJ (2015) Replication and inhibitors of enteroviruses and parechoviruses. Viruses 7: 4529-4562. doi: 10.3390/v7082832

van Ooij MJ, Glaudemans DH, Heus HA, van Kuppeveld FJ, Melchers WJ (2006a) Structural and functional integrity of the coxsackievirus B3 oriR: spacing between coaxial RNA helices. J Gen Virol 87: 689-695. doi: 10.1099/vir.0.81297-0

van Ooij MJ, Polacek C, Glaudemans DH, Kuijpers J, van Kuppeveld FJ, Andino R, Agol VI, Melchers WJ (2006b) Polyadenylation of genomic RNA and initiation of antigenomic RNA in a positivestrand RNA virus are controlled by the same cis-element. Nucleic Acids Res 34: 2953-2965. doi: 10.1093/nar/gkl349

van Ooij MJ, Vogt DA, Paul A, Castro C, Kuijpers J, van Kuppeveld FJ, Cameron CE, Wimmer E, Andino R, Melchers WJ (2006c) Structural and functional characterization of the coxsackievirus B3 CRE(2C): role of CRE(2C) in negative- and positive-strand RNA synthesis. J Gen Virol 87: 103-113. doi: 10.1099/vir.0.81297-0

Vogt DA, Andino R (2010) An RNA element at the 5'-end of the poliovirus genome functions as a general promoter for RNA synthesis. PLoS Pathog 6: e1000936. doi: 10.1371/journal.ppat.1000936

Witwer C, Rauscher S, Hofacker IL, Stadler PF (2001) Conserved RNA secondary structures in Picornaviridae genomes. Nucleic Acids Res 29: 5079-5089

Zoll J, Heus HA, van Kuppeveld FJ, Melchers WJ (2009) The structure-function relationship of the enterovirus 3'-UTR. Virus Res 139: 209-216. doi: 10.1016/j.virusres.2008.07.014 